\title{
The Role of Tumor Markers: Carcinoembryonic Antigen and Cancer Antigen 15-3 in Patients With Breast Cancer
}

Maimoona Khushk ${ }^{1}$, Adil Khan ${ }^{2}$, Abdur Rehman ${ }^{3}$, Sehrish Sheraz ${ }^{4}$, Yar Muhammad Tunio ${ }^{5}$, Kubra Rehman $^{6}$, Duaa Rehman ${ }^{7}$, Moiz Ahmed ${ }^{8,9}$, Kiran Abbas ${ }^{8}$, Muhammad E. Khan ${ }^{10}$

1. Department of Surgery, Peoples Medical College Hospital, Nawabshah, PAK 2. Department of Surgery, Shifa International Hospital, Islamabad, PAK 3. Department of Medicine, Usman Memorial Hospital, Karachi, PAK 4. Department of Public Health, Dow University of Health Sciences, Karachi, PAK 5. Department of Medicine, Gambat Institute of Medical Science, Khairpur, PAK 6. Department of Medicine, Shaheed Mohtarma Benazir Bhutto Medical College, Karachi, PAK 7. Department of Medicine, Liaquat National Hospital and Medical College, Karachi, PAK 8. Department of Medicine, Jinnah Postgraduate Medical Centre, Karachi, PAK 9. Department of Medicine and Surgery, Sindh Medical College, Karachi, PAK 10. Department of Oncology, Punjab Institute of Nuclear Medicine and Radiotherapy, Faisalabad, PAK

Corresponding author: Kiran Abbas, kiranabbas2020@gmail.com

\section{Abstract}

\section{Introduction}

Breast cancer is a major cause of mortality among females, worldwide. The present study was intended to evaluate the significance in the management of carcinoembryonic antigen (CEA) and cancer antigen 15-3 (CA15-3) in patients with breast cancer.

\section{Methodology}

A cohort study was conducted at the Jinnah Postgraduate Medical Center, Karachi, Pakistan from June 2020 to May 2021. All diagnosed cases of breast cancer who underwent surgical excision of tumor were eligible to partake. Patients who had metastatic breast cancer or had a recurrence were excluded. The patient's sociodemographic and clinical data were documented in a predefined pro forma. It included information about the age, sex, weight, as well as serum CEA and CA15-3. The CA15-3 and CEA levels for each patient were assessed by taking a $5 \mathrm{ml}$ blood sample and sending it to the laboratory for further workup. preoperatively on the second, seventh, and 28th postoperative days.

\section{Results}

A mean \pm SD age of $52.6 \pm 8.89$ years was reported. Family history of breast cancer was positive in one-fourth of the patients. Nodal metastasis was negative in $114(46.72 \%)$ patients. Three-fourth of patients had Stage II-IV with only a minority diagnosed with Stage I. The mean levels for CA15-3 in women with Stage I cancer was significantly lower on the seventh day and 28th postoperative day, compared to preoperative levels $(\mathrm{p}=$ 0.05). Similar associations were seen for stages II and III. Higher CEA levels were significantly associated with stage III breast cancer preoperatively $(5.88 \mathrm{ng} / \mathrm{ml}, \mathrm{p}=0.05)$ compared to postoperative values.

\section{Conclusion}

The current study revealed that preoperative values of serum CEA and CA15-3 significantly reduced postoperatively. Moreover, patients with advanced cancers had significantly higher levels of both tumor markers than those with less advanced diseases. The current study highlighted the importance of regular assessment of serum CEA and CA15-3 in breast cancer patients. Both these biomarkers are substantially elevated in breast cancer patients, preoperatively. Determining the levels of serum CEA and CA15-3 preand postoperatively may determine the prognosis and aid in forming the most optimal patient care regime with respect to the stage and subtype of cancer.

Categories: Internal Medicine, Oncology, Public Health

Keywords: ca 15-3, cea, breast cancer, cancer antigen 15-3, carcinoembryonic antigen

\section{Introduction}

The United States Centers for Disease Control (CDC) has reported breast cancer to be one of the major causes of death among females throughout the world [1]. Breast malignancy is diagnosed in over a million women each year and results in around four hundred thousand deaths [2-5].

In Pakistan, breast carcinomas consist of $14 \%$ of all tumors in women, thus are the most prevalent malignancy in the region [6]. in Karachi alone, Pakistan's largest city breast cancer comprises one-third of all 
tumors [7]. Survival in breast cancer is influenced by prompt diagnosis, appropriate treatment strategies, and genetic susceptibility. On the other hand, the prognosis is largely dependent on the histologic type of tumor, its grade, size, and distant metastasis. However, in many cases due to delayed presentation, lack of awareness, and lack of access to healthcare, by the time diagnosis is established, cancer has advanced to incurable stages.

Therefore, the identification of such tumor markers plays an important role in optimum cancer management. A patient's response to therapy and risk of recurrence can be assessed by regular monitoring of the tumor markers [8]. The use of carcinoembryonic antigen (CEA) and cancer antigen 15-3 (CA15-3) is not extensively explored in our setting. Therefore, the current study evaluated the prognostic role of pre- and post-operative levels of CEA and CA15-3 in the monitoring of disease activity in individuals who had breast cancer.

\section{Materials And Methods}

A cohort study was conducted at the Jinnah Postgraduate Medical Center, Sindh, Pakistan from June 2020 to May 2021, for a duration of 12 months. Ethical approval was obtained from the institute before data acquisition was started. A simple random sampling technique was used to select the patients using Google random number generator. The sample size was calculated using OpenEpi by keeping the likely proportion of $49.5 \%$, a confidence level of $95 \%$, and a margin of error of $6.27 \%$, a sample size of 244 was calculated. Patients were requested for informed written consent prior to participation after the aims and objectives were comprehensively narrated to them.

All histologically diagnosed cases of breast cancer who underwent surgical excision of tumor were eligible to partake. Patients who had metastatic breast cancer or had a recurrence were excluded. The patient's sociodemographic and clinical data were documented in a predefined pro forma. It included information about age, sex, weight, as well as serum CEA and CA15-3. The values for both the tumor markers for each patient were assessed preoperatively as well as at the second, seventh, and 28th day, postoperatively.

Statistical Package for the Social Sciences (SPSS version 24; IBM Corp., Armonk, NY) was used to perform data analysis. Chi-square test was applied to assess the correlation between the pre- and post-operative levels of tumor markers. A p-value of $<0.05$ was set as the cut-off value for statistical significance.

\section{Results}

A mean \pm SD age of $52.6 \pm 8.89$ years was reported. Family history of breast cancer was positive in 59 (24.18\%) of the patients. Nodal metastasis was negative in 114 (46.72\%) patients. A total of 173 (70.9\%) patients had advanced cancer (Stage II and III) with only a minority diagnosed with Stage I. The mean \pm SD level of cancer antigen (CA15-3) was $15.7 \pm 5.36 \mathrm{U} / \mathrm{ml}$ and of CEA was $1.7 \pm 1.1 \mathrm{ng} / \mathrm{ml}$. About 86 (35.25\%) patients had abnormal levels for CA15-3. Similarly, 45 (18.44\%) patients had abnormal CEA levels in our study (Table 1). 


\section{Cureus}

\section{Characteristics}

Age (mean $\pm S D)$

Family history

Yes

No

Clinical nodal status

NO

N1

N2

N3 and above

Clinical stage

I

II

III

Preoperative CA15-3 levels (mean \pm SD)

Normal $(<30 \mathrm{U} / \mathrm{ml})$

Abnormal (>30 U/ml)

Preoperative CEA levels (mean \pm SD)

Normal $(<5 \mathrm{ng} / \mathrm{ml})$

Abnormal ( $>5 \mathrm{ng} / \mathrm{ml})$
N (\%)

$52.6 \pm 8.89(32-68)$

$59(24.18 \%)$

$185(75.82 \%)$

$114(46.72 \%)$

$66(27.05 \%)$

$42(17.21 \%)$

$23(9.43 \%)$

$71(29.10 \%)$

$88(36.07 \%)$

$85(34.84 \%)$

$15.7 \pm 5.36(4.0-98)$

$158(64.75 \%)$

$86(35.25 \%)$

$1.7 \pm 1.1(0.6-41)$

$199(81.56 \%)$

$45(18.44 \%)$

TABLE 1: Clinical characteristics of the 244 patients.

CA15-3: cancer antigen 15-3; CEA: carcinoembryonic antigen.

The mean levels for CA15-3 in patients with Stage I cancer were significantly lower at postoperative seventh day and 28th day, compared to preoperative levels $(\mathrm{p}=0.05)$. Similar associations were seen for stages II and III. Higher CEA levels were significantly associated with stage III breast cancer preoperatively $(5.88 \mathrm{ng} / \mathrm{ml}, \mathrm{p}$ $=0.05$ ) compared to postoperative values (Table 2). 


\section{Cureus}

\begin{tabular}{|c|c|c|c|c|}
\hline \multirow[t]{2}{*}{ Patients (n) } & \multirow{2}{*}{$\begin{array}{l}\text { Preoperative } \\
\text { Mean }\end{array}$} & \multicolumn{3}{|c|}{ Postoperative (mean, p-value) } \\
\hline & & 2 days & 7 days & 28 days \\
\hline \multicolumn{5}{|c|}{ CA15-3 in U/mL } \\
\hline Stage I & 18.4 & 17.21 & $15.13^{\star}$ & $16.88^{*}$ \\
\hline Stage II & $31.9^{\star \star}$ & 32.43 & $16.92^{\star \star}$ & $22.33^{\star \star \star \star}$ \\
\hline Stage III & $47.2^{\star \star}$ & 47.11 & $19.96^{\star \star \star}$ & $34.98^{\star \star \star}$ \\
\hline \multicolumn{5}{|l|}{ CEA in ng/ml } \\
\hline Stage I & 1.95 & 2.11 & 2.01 & 1.69 \\
\hline Stage II & 2.69 & 2.86 & 2.62 & 2.22 \\
\hline Stage III & $5.88^{\star}$ & 5.79 & 5.71 & 3.96 \\
\hline
\end{tabular}

TABLE 2: Tumor biomarker levels in patients with breast cancer during follow-up, postoperatively.

${ }^{\star} p=0.05,{ }^{\star \star} p=0.01,{ }^{\star \star \star} p=0.001$. CA15-3: cancer antigen 15-3; CEA: carcinoembryonic antigen.

It was observed that above normal serum CEA and CA15-3 levels were more frequently reported in patients with advanced tumor stages (Table 3).

\begin{tabular}{|c|c|c|}
\hline Tumor biomarker & Stage & Frequency of abnormal levels \\
\hline \multirow[t]{3}{*}{ CA15-3 } & Stage I & $9.63 \%$ \\
\hline & Stage II & $51.06 \%$ \\
\hline & Stage III & $39.31 \%$ \\
\hline \multirow[t]{3}{*}{ CEA } & Stage I & $8.32 \%$ \\
\hline & Stage II & $31.30 \%$ \\
\hline & Stage III & $60.38 \%$ \\
\hline
\end{tabular}

TABLE 3: Frequency of abnormal levels of tumor biomarkers among patients according to the tumor stage.

CA15-3: cancer antigen 15-3; CEA: carcinoembryonic antigen.

\section{Discussion}

To monitor the activity of breast cancer tissue, certain biomarkers including CEA and CA15-3 play a vital role. The present study revealed that preoperative values of both aforementioned markers significantly differ postoperatively. Furthermore, patients with advanced cancers had significantly higher levels of CEA and CA15-3 than those with stage I, thus highlighting the importance of these serum markers for monitoring the progression of the tumors. Because the CEA and CA15-3 levels are elevated in patients preoperatively, therefore, they can be used as markers for breast cancer tissue activity.

Our findings were consistent with a study by Yin et al. who in 2018 identified an association between higher levels of tumor markers CEA and CA-153 in patients suffering from breast cancer [9]. A 2016 study by Fu et al. evaluated the significance of CA15-3 and CEA for establishing the prognosis of breast cancer. The results found a strong correlation between these markers and tumor stage [10]. Another study in 2019 revealed that patients who reported high levels of CA15-3 and CEA had a poorer prognosis compared to those with normal levels of CA15-3 and CEA [11]. Furthermore, the study concluded that the levels of CEA and CA15-3 were considerably associated with the luminal type of breast cancer.

Taghizadeh et al. in 2019 reported that $65.17 \%$ of the patients with breast cancer had elevated levels of 
serum CEA, while $57.29 \%$ of patients had higher CA15-3 levels. The levels of tumor markers had a strong correlation with the advanced stages of cancer [12]. Lian et al. in 2019 revealed similar findings, highlighting the role of tumor markers in breast cancer patients [13]. Based on the existing literature, serum CA15-3 can be utilized as a marker for advanced disease and even metastasis [14]. The preoperative serum levels of CA15-3 and CEA are shown to be elevated in advanced breast cancer disease with greater tumor size, lymph node metastasis, or aggressive histology. This indicates that raised tumor markers are associated with an increased tumor burden [15]. Both oncologists and surgeons are advised to check for serum CA15-3 which can help assess the need for an aggressive treatment protocol [16].

The current study had some limitations. Firstly, due to limited and undiversified sample size, the findings of the study cannot be generalized to a larger population. Further multi-center studies are needed to ascertain the role of these biomarkers as testing may increase overall healthcare expenses. Secondly, the follow-up period was very short hence, the long-term role of the CEA and CA15-3 should be explored in future studies.

\section{Conclusions}

The current study revealed that preoperative values of serum CEA and CA15-3 significantly reduced postoperatively. Moreover, patients with advanced cancers had significantly higher levels of both tumor markers than those with less advanced diseases. The current study highlighted the importance of regular assessment of serum CEA and CA15-3 - both are substantially elevated in breast cancer patients, preoperatively. Determining the levels of serum CEA and CA15-3 pre- and postoperatively may determine the prognosis and aid in forming the most optimal patient care regime with respect to the stage of cancer.

\section{Additional Information \\ Disclosures}

Human subjects: Consent was obtained or waived by all participants in this study. Jinnah Postgraduate Medical Centre issued approval IRB\#178-23-2020. This letter will serve as confirmation that the article entitled "The Role of Tumor Markers - Carcinoembryonic Antigen (CEA) and Cancer Antigen 15-3 (CA15-3) in Patients with Breast Cancer" has been reviewed and approved by the Institutional review board of Jinnah Postgraduate Medical Centre. . Animal subjects: All authors have confirmed that this study did not involve animal subjects or tissue. Conflicts of interest: In compliance with the ICMJE uniform disclosure form, all authors declare the following: Payment/services info: All authors have declared that no financial support was received from any organization for the submitted work. Financial relationships: All authors have declared that they have no financial relationships at present or within the previous three years with any organizations that might have an interest in the submitted work. Other relationships: All authors have declared that there are no other relationships or activities that could appear to have influenced the submitted work.

\section{References}

1. Miller JW, King JB, Joseph DA, Richardson LC, Centers for Disease Control and Prevention (CDC): Centers for Disease Control and Prevention (CDC). Breast cancer screening among adult women-behavioral risk factor surveillance system, United States, 2010. MMWR Morb Mortal Wkly Rep. 2012, 61:46-50.

2. Smigal C, Jemal A, Ward E, Cokkinides V, Smith R, Howe HL, Thun M: Trends in breast cancer by race and ethnicity: update 2006. CA Cancer J Clin. 2006, 56:168-83. 10.3322/canjclin.56.3.168

3. Boyle P, Ferlay J: Cancer incidence and mortality in Europe, 2004 . Ann Oncol. 2005, 16:481-8. 10.1093/annonc/mdi098

4. Hayat MJ, Howlader N, Reichman ME, Edwards BK: Cancer statistics, trends, and multiple primary cancer analyses from the Surveillance, Epidemiology, and End Results (SEER) Program. Oncologist. 2007, 12:20-37. 10.1634/theoncologist.12-1-20

5. Dikshit R, Gupta PC, Ramasundarahettige C, et al.: Cancer mortality in India: a nationally representative survey. Lancet. 2012, 379:1807-16. 10.1016/S0140-6736(12)60358-4

6. Qureshi MA, Mirza T, Khan S, et al.: Cancer patterns in Karachi (all districts), Pakistan: first results (20102015) from a pathology based cancer registry of the largest government-run diagnostic and reference center of Karachi. Cancer Epidemiol. 2016, 44:114-22. 10.1016/j.canep.2016.08.011

7. Bhurgri Y, Bhurgri A, Hassan SH, et al.: Cancer incidence in Karachi, Pakistan: first results from Karachi cancer registry. Int J Cancer. 2000, 85:325-9. 10.1002/(SICI)1097-0215(20000201)85:3<325::AIDIJC5>3.0.CO;2-J

8. Vizcarra E, Lluch A, Cibrián R, Jarque F, García-Conde J: CA 15.3, CEA and TPA tumor markers in the early diagnosis of breast cancer relapse. Oncology. 1994, 51:491-6. 10.1159/000227391

9. Yin WB, Yan MG, Fang X, Guo JJ, Xiong W, Zhang RP: Circulating circular RNA hsa_circ_0001785 acts as a diagnostic biomarker for breast cancer detection. Clin Chim Acta. 2018, 487:363-8. 10.1016/j.cca.2017.10.011

10. Fu Y, Li H: Assessing clinical significance of serum CA15-3 and carcinoembryonic antigen (CEA) levels in breast cancer patients: a meta-analysis. Med Sci Monit. 2016, 22:3154-62. 10.12659/msm.896563

11. Nam SE, Lim W, Jeong J, et al.: The prognostic significance of preoperative tumor marker (CEA, CA15-3) elevation in breast cancer patients: data from the Korean Breast Cancer Society Registry. Breast Cancer Res Treat. 2019, 177:669-78. 10.1007/s10549-019-05357-y

12. Taghizadeh A, Pourali L, Joudi M, et al.: Assessment of elevated serum tumor markers carcinoembryonic antigen (CEA) and cancer antigen 15-3 (CA15-3) among patients with different subtypes of metastatic 


\section{Cureus}

breast cancer. Middle East J Cancer. 2019, 10:17-22. 10.30476/MEJC.2019.44681

13. Lian M, Zhang C, Zhang D, et al.: The association of five preoperative serum tumor markers and pathological features in patients with breast cancer. J Clin Lab Anal. 2019, 33:e22875. 10.1002/jcla.22875

14. Valić A, Milas I, Mayer L, Šetić M, Matijević V, Stanec M.: Prognostic significance of CA 15-3 tumor marker in breast cancer patients. Libr Oncol: Croat J Oncol. 2017, 45:1-8.

15. Lee JS, Park S, Park JM, Cho JH, Kim SI, Park BW: Elevated levels of preoperative CA 15-3 and CEA serum levels have independently poor prognostic significance in breast cancer. Ann Oncol. 2013, 24:1225-31. 10.1093/annonc/mds604

16. Li J, Liu L, Feng Z, et al.: Tumor markers CA15-3, CA125, CEA and breast cancer survival by molecular subtype: a cohort study. Breast Cancer. 2020, 27:621-30. 10.1007/s12282-020-01058-3 